\title{
Facial emotion recognition in adolescents with personality pathology
}

\author{
Fleur Berenschot $\cdot$ Marcel A. G. van Aken • \\ Christel Hessels • Bram Orobio de Castro • \\ Ysbrand Pijl · Barbara Montagne · Guus van Voorst
}

Received: 11 November 2012 / Accepted: 10 October 2013/Published online: 29 October 2013

(c) Springer-Verlag Berlin Heidelberg 2013

\begin{abstract}
It has been argued that a heightened emotional sensitivity interferes with the cognitive processing of facial emotion recognition and may explain the intensified emotional reactions to external emotional stimuli of adults with personality pathology, such as borderline personality disorder (BPD). This study examines if and how deviations in facial emotion recognition also occur in adolescents with personality pathology. Forty-two adolescents with personality pathology, 111 healthy adolescents and 28 psychiatric adolescents without personality pathology completed the Emotion Recognition Task, measuring their accuracy and sensitivity in recognizing positive and negative emotion expressions presented in several, morphed, expression intensities. Adolescents with personality pathology showed an enhanced recognition accuracy of facial emotion expressions compared to healthy adolescents and clients with various Axis-I psychiatric diagnoses. They were also more sensitive to less intensive expressions of emotions
\end{abstract}

F. Berenschot - Y. Pijl - B. Montagne - G. van Voorst GGZ-Centraal Psychiatric Center, Amersfoort, The Netherlands

F. Berenschot

Triversum, Center for Child and Youth Psychiatry, Alkmaar,

The Netherlands

F. Berenschot ( $\square)$

Triversum, Kees Boekestraat 5, 1817 EZ Alkmaar,

The Netherlands

e-mail: fberenschot@ triversum.nl

M. A. G. van Aken - B. O. de Castro

Department of Developmental Psychology, Utrecht University, Utrecht, The Netherlands

M. A. G. van Aken - C. Hessels

Center for Adolescent Psychiatry, Reinier van Arkel group,

's-Hertogenbosch, The Netherlands than clients with various Axis-I psychiatric diagnoses, but not more than healthy adolescents. As has been shown in research on adults with BPD, adolescents with personality pathology show enhanced facial emotion recognition.

Keywords Facial emotion recognition · Personality pathology $\cdot$ Adolescents

\section{Introduction}

Every clinician who works with adolescents and adults with personality pathology is familiar with the problematic and complex social and emotional functioning of these clients. The heightened sensitivity, vulnerability and irascibility of these individuals can be a strenuous challenge in interpersonal interaction. Emotional dysregulation, instability and impulsivity, and disturbed social functioning are regarded as the central characteristics of personality pathology, particularly of the borderline personality disorder (BPD) [1].

Deviant and disturbed social cognition is seen as one of the core mechanisms underlying the development and maintenance of BPD [2,3]. Clinicians and researchers share a common hypothesis that individuals with BPD do not adequately process and appraise emotional and social information of self and others.

One fundamental aspect of interpersonal functioning is the ability to recognize and understand social signals, such as emotion expressions [4]. The ability to recognize and interpret the emotional states of others can be considered a cognitive basis of intact social functioning, with facial emotion recognition as a cognitive cornerstone [5]. Disturbances in facial emotion recognition have been examined in adults, with regard to several psychiatric conditions, especially autism, schizophrenia, depression and psychosis. 
A relatively small degree of research was directed specifically at facial emotion recognition in personality pathology, mostly in anti-social personality disorder and BPD. In some research static images of emotional facial expressions were used. In other studies images of facial affect, which were electronically morphed from a neutral expression to emotional expressions with increasing intensity, were used to examine recognition and detection threshold simultaneously (for a review see Domes et al. [5]). The studies reveal a pattern of alterations in facial emotion recognition in individuals with BPD. Results were, however, mixed and sometimes contradictory, with both enhanced and impaired emotion recognition [6-9]. The latest findings suggest that the abnormalities in processing basic facial emotions in adults with BPD are subtle and specific, with a heightened sensitivity or hypersensitivity (i.e., lower detection threshold) for negative emotions and a negativity or anger bias in the evaluation of ambiguous, or neutral facial expressions [3, 5, 10]. Domes et al. [5] suggest that emotional hyperreactivity (intensified emotional reactions to external emotional stimuli) interferes with the cognitive processes of facial emotion recognition.

It is important to not only identify cognitive impairments in personality disorders, but to also search for the earlier phases of development in which impairments and deviations may be found as a concomitant of personality disorders [11]. However, research into specific cognitive processing of social information in children and adolescents with personality pathology is sparse. This may be due to the fact that the diagnosis of personality disorders in adolescence has long been controversial because of the perceived instability of personality in adolescence, the stigma associated with this diagnosis and a tendency to explain Axis-II symptoms with Axis-I symptoms [12, 13]. By now it has become increasingly evident that personality disorders can be reliably and validly diagnosed in adolescence and that personality pathology is an important and serious form of psychopathology, also in this stage of development [12-15]. Clinicians and researchers therefore state that it is crucial that personality pathology is acknowledged and detected in adolescence and distinguished from Axis-I disorders [12]. Better understanding of personality pathology in adolescence could lead to the elaboration of specific interventions in this critical developmental phase that may prevent deterioration in psychosocial functioning and promote early and faster recovery [14, 15]. Research into social information processing in adolescents with personality pathology is especially important considering the fact that adolescence is a highly critical phase in emotional and social development. The question is relevant whether deficits in social information processing already exist in the early stages of personality disorder development, and if so, what the best intervention method is in this phase [16].

We found two recent relevant studies on social information processing in adolescents with personality pathology. One study analyzed distortions in attention maintenance of positive, negative or neutral emotional stimuli in adolescents with BPD and found an attention bias for negative emotional stimuli when clients were in a negative mood [17]. Sharp et al. [13] recently found that hypermentalizing (overinterpretative mental state reasoning), instead of a break-down of mentalizing, was strongly associated with BPD features in adolescents which means that adolescents with BPD features tended to overinterpret social signs. To our knowledge, no studies specifically investigated facial emotion recognition in adolescents with personality pathology.

We mainly discuss studies of adults with BPD because most studies of facial emotion recognition in personality pathology with comparable techniques have been performed in adults with BPD and because Cluster-B pathology seems to be most prominent in our adolescent sample. However, in the present study, we decided to focus on broader personality pathology and not specifically on BPD alone, because it is known that in adolescents features of different personality disorders can still coincide and overlap, also in accordance with the recent dimensional approach of personality disorders [12, 18].

Aim of the present study was to investigate the recognition of facial expressions of emotions presented in several, morphed, expression intensities in adolescents with personality pathology in comparison with a healthy adolescent group. An adolescent client group with various Axis-I psychiatric diagnoses, without an Axis-II classification was added as a comparison group to distinguish that hypothesized alterations in emotion recognition in the personality pathology group are not due to the comorbid axis-I pathology of this group.

Based on research in adult personality disorders, it was hypothesized that adolescent clients with personality pathology are more accurate and more sensitive (i.e., have lower threshold levels for recognition) in recognizing facial emotions, in particular negative emotions, than healthy controls and adolescents with other psychopathology.

\section{Method}

Participants and setting

In the present study, a group of adolescents with personality pathology and comorbid Axis-I psychiatric diagnoses was compared with a group of healthy adolescents and a 
group of adolescents with various Axis-I psychiatric diagnoses, without personality pathology.

The group of adolescents with personality pathology consisted of 42 clients ( 34 girls, 8 boys; 12 in-patients, 30 out-patients). They ranged in age between 12 and 18, and were recruited at three locations of a specialized center for child and adolescent psychiatry in The Netherlands. This center treats clients with moderate to severe psychiatric pathology, who have impaired functioning in several domains (school, family and social relations). All clients that met the inclusion criteria of a diagnosed personality disorder not otherwise specified $(n=25)$, or deferred diagnosis on Axis-II $(n=18)$, according to the DSM-IVTR diagnostic criteria (APA [1]), were included. Seven more clients were tested but excluded from the study because an Axis-II disorder was ultimately not diagnosed $(n=3)$, or their total IQ score was less than $85(n=4)$. Medication use, schizophrenia or pervasive development disorders were also exclusion criteria for this study. None of the patients had to be excluded because of these exclusion criteria.

Comorbid Axis-I diagnoses in this group included internalizing disorders $(n=23)$ (anxiety disorder, specific phobia, adjustment disorder with mixed anxiety and depressed mood, dysthymic disorder, depressive disorder, identity problem, eating disorder, PTSS), disruptive behavior disorders $(n=10)$ (attention deficit disorder, behavioral disorder NOS), and other disorders $(n=9)$ (parent-child relational problems, disorder of adolescence NOS, no diagnosis on Axis-I). Sixty-four percent of the clients had more than one diagnosis on Axis-I and the mean global assessment of functioning $(\mathrm{GAF})$ was $54(\mathrm{SD}=6)$.

The DSM-IV-TR, Axis-II diagnoses were the result of consensus-oriented multi-disciplinary team decision-making, following an extensive diagnostic evaluation phase including separate interviews by a child-psychiatrist and a clinical psychologist, (neuro)psychological assessment and a family interview. In this department of child and adolescent psychiatry it is common policy to administer a DSM-IV-TR, Axis-II classification; not otherwise specified, or delayed, and not a specific personality disorder when the client is under 18 years.

To gain insight into the type of personality pathology within this patient group, clinicians assessed the nature of Axis-II symptoms on five-point rating scales, ranging from clearly absent to present, in an Axis-II questionnaire in which the items reflected all the DSM-IV-TR criteria for personality disorders. Visual inspection revealed that of the 42 adolescents, 25 participants had the highest mean score on Cluster-B pathology, 13 participants had the highest mean score on Cluster-C pathology, and 4 participants on Cluster-A pathology. Six participants that scored highest on Cluster-C pathology also had a high mean score $(\geq 3)$ on the borderline personality disorder subscale.

The non-referred control group of 111 healthy adolescents (54 boys, 57 girls) was recruited from a secondary school. They ranged in age between 12 and 18. These adolescents never had psychological or psychiatric treatment. The students were asked in writing if they ever had psychological counseling/therapy. Two persons answered yes to this question and were excluded.

The psychiatric comparison group consisted of 28 clients (13 girls, 15 boys; all out-patients) with various Axis-I disorders, but no diagnosis on Axis-II, based on the abovementioned diagnostic procedure in the multi-disciplinary team. They ranged in age between 12 and 18. These adolescents were tested as part of a diagnostic neuropsychological assessment in the same center as the group with personality pathology. Seven clients met criteria for internalizing disorders (anxiety disorder, depressive disorder, obsessive-compulsive disorder), 16 for disruptive behavior disorders (attention deficit disorder, opposition defiant disorder), and five for other disorders (disorder of adolescence NOS, expressive language disorder).

Information about the intelligence (IQ) of the two client groups was gathered from the client files. The clients' intelligence profiles from the Dutch translation of the Wechsler Intelligence Scales for Children (WISC-III ${ }^{\mathrm{NL}}$ ) and the Dutch translation of the Wechsler Adult Intelligence Scales (WAIS) were not older than 2 years. Total IQ, verbal IQ and performance IQ were average in both patient groups and did not differ significantly.

The indicator for cognitive functioning in the healthy adolescents group was the current education level. A majority of $52.3 \%$ was in the average general secondary education level and 21.6 and $26.1 \%$, respectively, were in lower general secondary education and pre-university education. In view of the education level of the healthy adolescent group significant deviations of the average IQ in each group from 100 were deemed unlikely.

The personality pathology group proved significantly older than the control groups, as is shown in Table 1. The high number of girls in the personality pathology group reflects that personality disorders are more often diagnosed in women than in men. Group numbers were unequal because we did not sample from the population of the child and adolescent center (all eligible patients were invited to participate) and sampled classes for the secondary school. The use of existing groups meant that sex and age distribution could not be controlled in the sampling design when including participants in the study. Because of the expected effect of these factors on emotion recognition, age and sex were controlled statistically in the main analyses. 
Table 1 Subject characteristics

\begin{tabular}{|c|c|c|c|c|}
\hline & $\begin{array}{l}\text { Clients with personality } \\
\text { pathology }\end{array}$ & $\begin{array}{l}\text { Healthy } \\
\text { controls }\end{array}$ & $\begin{array}{l}\text { Clients with various Axis-I } \\
\text { psychiatric diagnoses }\end{array}$ & $\begin{array}{l}\text { Association } \\
\text { with group }\end{array}$ \\
\hline$n$ & 42 & 111 & 28 & \\
\hline \multicolumn{5}{|l|}{ Sex } \\
\hline$n(\%)$ boys & $8(19 \%)$ & $54(49 \%)$ & $15(54 \%)$ & $\chi^{2}(6)=24.8$ \\
\hline$n(\%)$ girls & $34(81 \%)$ & $57(51 \%)$ & $13(46 \%)$ & $p<0.00$ \\
\hline \multicolumn{5}{|l|}{ Age } \\
\hline Mean & 15.7 & 14.4 & 14.0 & $F(2,178)=15.0$ \\
\hline SD & 1.4 & 1.6 & 1.4 & $p<0.00$ \\
\hline \multicolumn{5}{|l|}{ IQ } \\
\hline Mean & 102.7 & & 101.4 & $F(1,68)=0.23$ \\
\hline SD & 11.8 & & 10.2 & $p=0.63$ \\
\hline
\end{tabular}

\section{Procedure}

Participants of the two client groups were tested with an emotion recognition task as part of a more extensive psychological assessment of emotional functioning and psychopathology, consisting of several questionnaires and (neuro)psychological computer tests. First the purpose of the assessment was explained. During testing the examiner remained in the room and participants were invited to ask questions in case directions were unclear. Participants were seated in front of the computer and asked to perform the emotion recognition task. Three practice trials preceded the actual task. Participants had to indicate which emotion they perceived from a set of six possible emotion labels (i.e., forced choice). Participants of the healthy control group were tested in a quiet test room, in small groups of approximately 10-16 people per session. Explanation was offered group wise. The researcher was available for extra explanation.

The study was approved by the 'Science Commission' of GGZ-Centraal psychiatric center and was conducted in accordance with the local ethical standards. In both patient groups participants and their parents/caretakers, if participants were under age 16, signed informed consent forms. The consent procedure for the healthy group was that the adolescents of six classes with different education levels were invited to participate by means of a letter addressed to their parents with a written explanation of the study. A passive consent procedure was applied (as approved by the school board), in which parents were asked to give notice in case they did not want their child to participate. One student's parents did so.

\section{Measures}

\section{Emotion recognition task (ERT)}

In this study an emotion recognition task with morphing technique was used to be able to measure not only

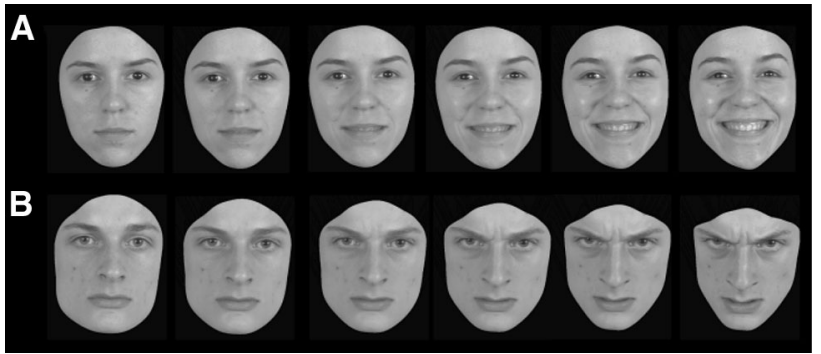

Fig. 1 The emotional recognition task. a Shows the gradual change from neutral $(0 \%)$ to happiness $(100 \%)$ in steps of $20 \%$. b Shows the gradual change from neutral $(0 \%)$ to anger $(100 \%)$ in steps of $20 \%$. The actual increments were steps of $10 \%$, starting at $20 \%$

recognition at $100 \%$ facial expression, but also the detection threshold. The emotional facial stimuli used in this study were developed by Montagne et al. [19] and are based on colored pictures $(11.2 \times 11.7 \mathrm{~cm})$ of actors mimicking emotional expressions. A computer-generated program, developed from algorithms designed by Benson and Perrett [20], was used to create morphed photo video clips of emotions in intermediate intensities. In these clips a neutral face ( $0 \%$ emotion expression) morphs into a fullblown emotion expression $(100 \%)$. A set of nine (expression intensity: 20, 30, 40, 50, 60, 70, 80, 90, $100 \%) \times 6$ (emotions: anger, disgust, fear, sadness, happiness and surprise) $\times 4$ actors (two male, two female) stimuli constituted the stimuli material $(=216$ stimuli) (Fig. 1).

In the practice trials, the participant saw in a random order three photo-sequences of three emotional expressions of two actors, running from 0 to $100 \%$ expression intensity. After that the actual test started. The participants saw all photo-sequences of the four actors and six emotions (in random order) in nine sets of expression intensity, running from 0 to $20 \%$ expression, subsequently photo-sequences from 0 to $30 \%$, from 0 to $40 \%$, etc., until they reached the final sequence of photos in which the neutral face morphed into full-blown, $100 \%$ expression intensities. After each 
trial, the participant was required to make a forced choice between one of six emotional expression labels that were displayed on the left-hand side of the screen. The animation remained on the screen until the participant gave a response. There was no time restriction. The duration of the photo-sequences varied from $0.5 \mathrm{~s}(20 \%$ expression intensity) to $2 \mathrm{~s}$ ( $100 \%$ expression intensity).

Two types of dependent measures were recorded: accuracy and sensitivity. Accuracy was operationalized as the percentage of correctly identified emotional expressions of the $100 \%$ expression intensity sequence sets. Sensitivity was operationalized as the minimum amount of expression required for systematic correct identification; more specifically, the minimum amount of emotion expression intensity from which point onwards the expression was consistently recognized in subsequent expression intensity trials. This procedure was selected to ensure that participants actually recognized the emotional facial expression, rather than producing chance 'hits'. In case participants failed to recognize subsequent expression intensities of an emotion at each of the four actor photosequences, their sensitivity scores for that specific emotion were attributed the highest possible expression intensity, viz. $100 \%$.

\section{Data analysis}

A multivariate analysis of (co)variance was conducted using a general linear model (GLM in SPSS18) in order to test the predicted differences. The within-subjects design consisted of one two-level factor emotion valence (negative emotions: anger, sadness, fear, disgust, vs. positive emotions; happiness, surprise) and the two dependent variables accuracy and sensitivity in recognizing emotions. The three levels of the between-subjects factor group were the group of participants with personality pathology, healthy adolescent controls and clients with various Axis-I psychiatric disorders. The covariate age and the fixed factor sex were added to the between-subjects design as control variables. The design is unbalanced because of the associations between the control variables and group. Both the method of partitioning the total sums of squares and the choice of effects in the model and possibly even the order of effects in the model will therefore affect the results. For that reason, the weighted squares of means technique (Type III sums of squares) was used to obtain conservative tests of group effects adjusted for all other effects in the model. This statistical model enabled us to test the hypothesized superior accuracy and sensitivity of adolescents with personality pathology in recognizing emotions in general and negative emotions in particular by testing the main effects and related simple contrasts for the personality pathology group versus each control group as well as the test of the interaction between this group factor and valence of emotion.

\section{Results}

Preliminary data analyses

Before testing any model, the homogeneity of regression assumption was tested. A model containing the interactions between the covariate Age and the factors Sex and Group was specified. The smallest $p$ value was $p=0.22$ for the three-way interaction between Sex, Age and Group (Wilks' Lambda $=0.966, F(4,336)=1.5)$. Therefore, we removed this three-way interaction and the interactions between covariate and between-subjects factors from the model. The mixed mancova multivariate test results are shown in Table 2.

Girls were both more accurate and sensitive interpreters of the facial expression of emotion than boys. A weak relation was found between age and accuracy, and age and sensitivity. Older adolescents performed better.

Main analyses

The main Group effect proved medium size (partial $\eta^{2}=0.06$ ) and significant at the 0.05 level. The univariate tests of the Group main effect were significant for both accuracy and sensitivity, see Table 3. Simple contrast with the personality pathology group as the reference group showed that the personality pathology group was more accurate, $F(1,174)=5.1, p=0.03$, but equally sensitive, $F(1,174)=0.2, p=0.66$, than the healthy controls. The personality pathology group was more accurate,

Table 2 Mixed mancova multivariate tests

\begin{tabular}{llllll}
\hline Effect & $\begin{array}{l}\text { Wilks' } \\
\Lambda\end{array}$ & $F$ & $d f 1$ & $d f 2$ & $p$ \\
& & & & & \\
\hline Between subjects & 0.944 & 5.1 & 2 & 173 & 0.01 \\
$\quad$ Age & 0.889 & 10.8 & 2 & 173 & 0.00 \\
Sex & 0.879 & 5.7 & 4 & 346 & 0.00 \\
Group & 0.956 & 2.0 & 4 & 346 & 0.10 \\
Sex $\times$ group & & & & & \\
Within subjects & 0.985 & 1.3 & 2 & 173 & 0.27 \\
Emotion valence & 0.999 & 0.1 & 2 & 173 & 0.95 \\
Emotion valence $\times$ age & 0.990 & 0.8 & 2 & 173 & 0.43 \\
Emotion valence $\times$ sex & 0.959 & 1.9 & 4 & 346 & 0.12 \\
Emotion valence $\times$ group & 0.991 & 0.4 & 4 & 346 & 0.82 \\
Emotion & & & & & \\
valence $\times$ sex $\times$ group & & & & &
\end{tabular}


Table 3 Group main effect: estimated means (unweighted means for average age $=14.6$ years) and univariate tests

\begin{tabular}{|c|c|c|c|c|c|c|c|c|c|c|}
\hline \multirow[t]{2}{*}{ Emotion } & \multicolumn{3}{|c|}{$\begin{array}{l}\text { Clients with personality } \\
\text { pathology }(n=42)\end{array}$} & \multicolumn{3}{|c|}{$\begin{array}{l}\text { Healthy controls } \\
(n=111)\end{array}$} & \multicolumn{3}{|c|}{$\begin{array}{l}\text { Clients with various Axis-I } \\
\text { psychiatric diagnoses } \\
(n=28)\end{array}$} & \multirow[t]{2}{*}{$\begin{array}{l}\text { Univariate test of main } \\
\text { effect all emotions }\end{array}$} \\
\hline & $M$ & SE & $95 \% \mathrm{CI}$ & $M$ & SE & $95 \% \mathrm{CI}$ & $M$ & SE & $95 \% \mathrm{CI}$ & \\
\hline \multicolumn{11}{|l|}{ Accuracy $^{\mathrm{a}}$} \\
\hline All & 78.8 & 2.1 & $75-83$ & 73.6 & 1.0 & $72-76$ & 70.9 & 1.9 & $67-75$ & \multirow[t]{3}{*}{$F(2,174)=3.9, p=0.02$} \\
\hline Negative & 76.5 & 2.9 & $71-82$ & 67.1 & 1.3 & $64-70$ & 65.9 & 2.7 & $61-71$ & \\
\hline Positive & 81.1 & 2.4 & $76-86$ & 80.1 & 1.1 & $78-82$ & 75.9 & 2.2 & $71-80$ & \\
\hline \multicolumn{11}{|l|}{ Sensitivity $^{\mathrm{b}}$} \\
\hline All & 54.3 & 1.7 & $51-58$ & 55.1 & 0.8 & $54-57$ & 62.5 & 1.6 & $59-66$ & \multirow[t]{3}{*}{$F(2,174)=9.0, p=0.00$} \\
\hline Negative & 62.3 & 2.1 & $58-66$ & 65.2 & 0.98 & $63-67$ & 71.2 & 2.0 & $67-75$ & \\
\hline Positive & 46.2 & 2.4 & $42-51$ & 45.0 & 0.11 & $43-47$ & 53.8 & 2.2 & $49-58$ & \\
\hline
\end{tabular}

${ }^{a}$ Means reflect the percentage of correct responses at $100 \%$ emotion expression intensity

${ }^{\mathrm{b}}$ Means reflect the minimal percentage of emotion expression intensity needed for recognition

\section{Accuracy}

Personality pathology $\llbracket$ Healthy controls $\square$ Various Axis-I psychiatric diagnose

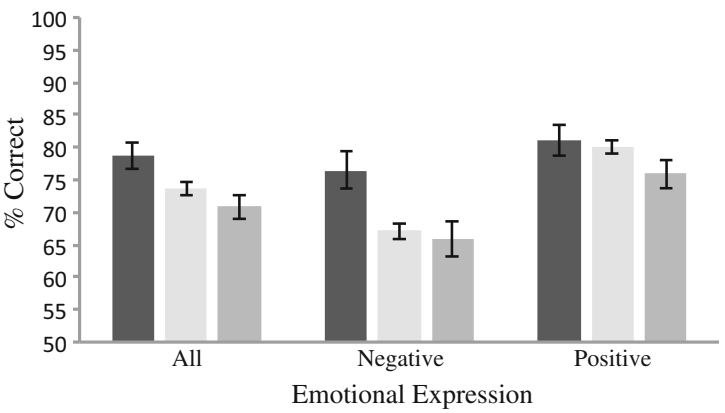

Fig. 2 Accuracy: group differences in mean percentage of correct responses at $100 \%$ facial emotion expression intensity. Error bars represent standard error of the mean

Sensitivity

- Personality pathology ${ }^{\circledR}$ Healthy controls $₫$ Various Axis-I psychiatric diagnoses

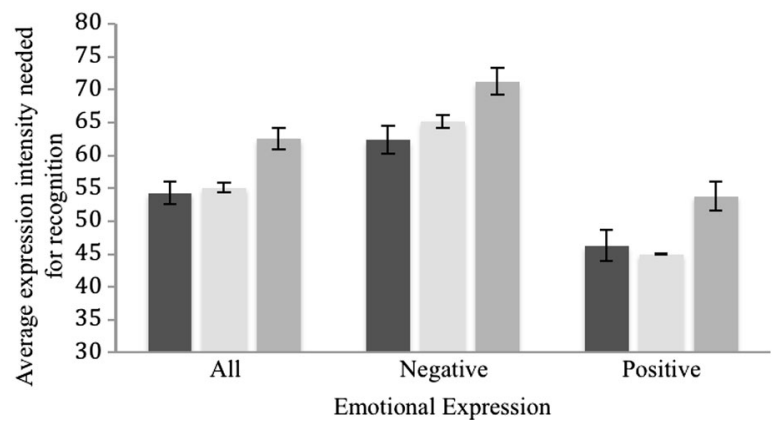

Fig. 3 Sensitivity: group differences in mean minimal facial emotion expression intensity needed for recognition. Low values reflect less expression intensity needed and therefore higher sensitivity. Error bars represent standard error of the mean.

$F(1,174)=7.5, \quad p=0.01, \quad$ and more sensitive, $F(1,174)=11.5, p<0.01$, than the controls with various Axis-I psychiatric disorders.
The Emotion valence by Group interaction had a small to medium effect, partial $\eta^{2}=0.02$. It was entirely due to the superior accuracy of the personality pathology group in recognizing negative emotions, as expected. The differences between groups in accuracy of recognizing positive emotions followed the same pattern, but were smaller (Fig. 2). Yet, this Emotion valence by Group interaction was not significant at an acceptable level, either in the multivariate test (Table $2 ; p=0.12$ ) or in corresponding univariate tests [Accuracy: $F(2,174)=2.8, p=0.06$; Sensitivity: $F(2,174)=1.1, p=0.33]$.

In sum, the hypothesis with regard to superior accuracy of adolescents with personality pathology in recognizing emotions was supported. The hypothesis of enhanced sensitivity of the personality pathology group was only partly supported (Fig. 3). There was a difference in sensitivity between the personality pathology group and the clients with various Axis-I psychiatric pathology, but not in comparison to the healthy control group. The hypothesis that adolescent clients with personality pathology are better in recognizing negative, rather than positive emotions was not supported.

\section{Discussion}

The aim of this study was to examine recognition of facial emotion expressions in adolescents with personality pathology. Results showed an enhanced recognition accuracy of facial emotion expressions in adolescents with personality pathology compared to healthy controls and patients with various psychiatric diagnoses. The hypotheses regarding a lower detection threshold in recognizing emotions, and superior recognition of specifically negative emotions, were partly confirmed. 
At full emotion expression intensity adolescents with personality pathology were more accurate than both control groups in recognizing facial emotion expressions. Regarding sensitivity (i.e., detection threshold for emotion recognition) results showed that as faces morphed from neutral to maximum emotional expression intensity, the adolescents with personality pathology generally recognized facial emotion expressions at an earlier stage (i.e., needing less facial emotion expression intensity for adequate recognition) than the psychiatric control group, but not earlier than the healthy adolescents. The study results could not support the hypothesis that the adolescents with personality pathology were better in accurately recognizing negative emotions in particular. To our best knowledge, this is the first study in which facial emotion recognition in adolescents with personality pathology was examined and in which facial morphing technique for this specific group was used.

There are no comparable studies of facial emotion recognition in adolescents with personality pathology, but our results correspond to previous research in adults with BPD in which heightened recognition of facial or eye region emotion expression was found $[3,8,21]$. Our findings are inconsistent with earlier studies using static emotional facial expressions that showed that adults with BPD have impaired emotion recognition [6, 7]. Two previous studies used facial morphing techniques with contradictory results regarding sensitivity $[8,22]$. In our study a lower detection threshold for emotion recognition was only found compared to psychiatric controls, but not to healthy controls, which is consistent with the study of Domes et al. [22]. Studies that did find a heightened sensitivity or response bias for negative emotions generally used neutral or ambiguous stimuli and/or results were emotion specific (anger and fear) [22, 23]. In the current study, a $20 \%$ emotion expression was the lowest expression intensity so there were no completely neutral stimuli. Also we did no analysis on the level of single emotions, which could explain why these hypotheses were not confirmed.

The findings of this study complement the previous findings in adults, namely that the enhanced facial emotion recognition is already present when personality pathology is manifest at an earlier age, i.e., in adolescence.

What could explain this enhanced facial emotion recognition and how does it relate to the apparently paradoxical impaired interpersonal functioning? The enhanced facial emotion recognition in the personality pathology group, found in this study is in line with theories about hypersensitivity in personality pathology. Von Ceumern-Lindenstjerna et al. [17] showed an attention bias for negative facial expressions in female adolescents with BPD. Possibly, this attention bias coincides with an increased vigilance for emotions based on previous negative experiences and underlying feelings and cognitions of unsafety and distrust, anticipating potential rejection and emotional distress. Internal attachment and attribution style, cognitive bias and contextual factors may play an important part in this increased vigilance and hypersensitivity $[9,24,25]$. The hypersensitivity and tendency to constantly check other persons' emotions based on their facial expressions could hypothetically be a constant training in reading facial emotions, thereby enhancing emotion recognition. However, an enhanced ability to recognize facial emotion expressions does not seem to be an advantage. It may cause individuals with personality pathology to experience more emotions and trigger negative and hostile cognitions which, in combination with their problems with inhibitory functions and emotion regulation, could lead them to overreact, even in minor emotional events [4, 5, 8, 14, 23, 26, 27]. Sharp et al. [13] describe this process as hypermentalizing. The adolescent with BPD seems to overinterpret social cues and what others are thinking and seems to be unable to regulate the anxious rumination caused by this overinterpretation. The vicious circle of emotional overinterpretation and dysregulation that follows the enhanced emotion recognition causes negative experiences in social contacts. This study shows that adolescents with personality pathology have this enhanced emotion recognition in a phase in which peer relations are crucial, social skills are in development and the adolescent is extra vulnerable to negative experiences in social interaction.

There are some limitations to this study. The first limitation to our study is that, because of limited time and financial restrictions, standardized diagnostic instruments could not be administered to assess the personality pathology diagnoses and comorbidity. The mean scores of the Axis-II questionnaire point out that Cluster-B pathology, and more specifically borderline personality pathology, in the client group seems to be most prominent. However, we cannot state this unequivocally without structured diagnostic measurement. Future studies could aim for more standardized diagnostic inclusion criteria. Moreover, it could be valuable to not only take a categorical point of view, but also a dimensional starting point to study personality pathology traits, the severity thereof and the relationship of severity and type of personality pathology with facial emotion recognition.

Another limitation concerns the measurement of the dependent variables. Although the morphing technique seems to be more dynamic than the use of static stimuli, the test situation still does not fully resemble real-life experiences with greater time pressure and, possibly, conditions of heightened emotional states. Speed of processing (reaction time), attention, impulse control, visual perception, verbal and non-verbal intelligence and the use of multimodal and neutral stimuli and a free-choice format are variables and factors that might increase the external validity of future research. 
The study design was perhaps not optimal, because we had to work with existing clinical groups. An age and sexstratified sample was not an option. Controlling variables in the sample design (instead of in the statistical analyses) could have allowed for equal cell frequencies and more powerful tests.

In conclusion, our study is the first to reveal enhanced recognition of facial emotion expression in adolescents with personality pathology compared to healthy adolescents and adolescents with other psychopathology. It shows that the enhanced recognition that seems to be present in adults with BPD also exists in younger clients with personality pathology. Possibly, the difficulties and stress that adolescents with personality pathology experience in social interaction are not due to impaired emotion recognition, but rather to a better ability at identifying facial emotion expression and possibly, the hypermentalizing that ensues. This enhanced recognition could play a part in developing and/or maintaining the impairments in social functioning of this vulnerable group. If so, this would have important implications for therapeutic (early) intervention and theory. Therapists should be aware of the enhanced ability of their young clients to recognize facial emotion expressions. Therapeutic interventions should focus on teaching the adolescents to interpret the perceived emotion of other people in adequate meaning and regulate their own emotions and interpretations of the mental state of the other person. Further studies addressing the neurobiological, neurocognitive and psychosocial factors involved are necessary to shed further light on social information processing in early personality pathology.

\section{Conflict of interest None.}

\section{References}

1. American Psychiatric Association (2000) Diagnostic and statistical manual of mental disorders, fourth edition, test revision. American Psychiatric association, Washington DC

2. Fonagy P, Bateman A (2008) The development of borderline personality disorder - a mentalizing model. J Pers Disord 22(1):4-21

3. Scott LN, Levy KN, Adams RB, Stevenson MT (2011) Mental state decoding abilities in young adults with borderline personality disorder traits. Personal Disord: Theory, Res Treat 2:98-112

4. Minzenberg MJ, Poole JH, Vinogrodov S (2006) Social-emotion recognition in borderline personality disorder. Compr Psychiatry 47:468-474

5. Domes G, Schulze L, Herpertz SC (2009) Emotion recognition in borderline personality disorder: a review of the literature. J Pers Disord 23:6-19

6. Bland AR, Williams CA, Scharer K, Manning S (2004) Emotion processing in borderline personality disorders. Issues Ment Health Nurs 25:655-672

7. Levine D, Marziali E, Hood J (1997) Emotion processing in borderline personality disorders. J Nerv Ment Dis 186:240-246
8. Lynch RT, Rosenthal MZ, Kosson DS, Cheavens JS, Lejuez CW, Blair RJR (2006) Heightened sensitivity tot facial expression of emotion in borderline personality disorder. Emotion 6:645-655

9. Wagner AW, Linehan MM (1999) Facial expression recognition ability among women with borderline personality disorder: implications for emotion regulation? J Pers Disord 13:329-344

10. Dijck M, Habel U, Slodczyk J, Schlummer J, Backes V, Schneider F, Reske M (2009) Negative bias in fast emotion discrimination in borderline personality disorder. Psychol Med 39:855-864

11. Judd PH (2005) Neurocognitive impairment as moderator in the development of borderline personality disorder. Dev Psychopathol 17:1173-1196

12. Miller AL, Muehlenkamp JJ, Jacobson CM (2008) Fact or fiction: diagnosing borderline personality disorder in adolescents. Clin Psychol Rev 28:969-981

13. Sharp C, Pane H, Ha C, Venta A, Patel AB, Sturek J, Fonagy P (2011) Theory of mind and emotion regulation difficulties in adolescents with borderline traits. J Am Acad Child Adolesc Psychiatry 50(6):563-573

14. Bleiberg E (2001) Treating personality disorders in children and adolescents, a relational approach. The Guilford Press, New York

15. Chanen AM, Jovey MJ, Jackson HJ (2007) Adaptive functioning and psychiatric symptoms in adolescents with borderline personality disorder. J Clin Psychiatry 68:297-306

16. Gratz KL, Tull MT, Reynolds EK, Bagge CL, Latzman RD, Daughters SB, Lejeuz CW (2009) Extending extant models of the pathogenesis of borderline personality disorder to childhood borderline personality symptoms: the role of affective dysfunction, disinhibition, and self- and emotion regulation deficits. Dev Psychopathol 21:1263-1291

17. Von Ceumern-Lindenstjerna I, Brunner R, Parzer P, Mundt C, Fiedler P, Resch F (2010) Attentional bias in later stages of emotional information processing in female adolescents with borderline personality disorder. Psychopathology 43:25-32

18. Morey LC, Berghuis H, Bender DS, Verheul R, Krueger RF, Skodol AE (2011) Toward a model for assessing level of personality functioning in DSM-5, Part II: empirical articulation of a core dimension of personality pathology. J Pers Assess 93(4):347-353

19. Montagne B, Kessels RPC, De Haan EHF, Perrett DI (2007) The emotion recognition task: a new paradigm to study the perception of facial emotional expressions at different intensities. Percept Mot Skills 104:589-598

20. Benson PJ, Perrett DI (1991) Synthesising continuous-tone caricatures. Image Vis Comput 9:123-129

21. Fertuck EA, Jekal A, Song I, Wyman B, Morris MC, Wilson ST, Stanley BS (2009) Enhanced "reading the mind in the eyes" in borderline personality disorder compared to healthy controls. Psychol Med 39:1979-1988

22. Domes G, Czieschnek D, Weidler F, Berger C, Fast K, Herpertz S (2008) Recognition of facial affect in borderline personality disorder. J Pers Disord 22(2):135-147

23. Donegan NH, Sanislow CA, Blumberg HP, Fulbright RK, Lacadie C, Skudlarski P, Wexler BE (2003) Amygdala hyperreactivity in borderline personality disorder: implications for emotional dysregulation. Biol Psychiatry 54:1284-1293

24. Bartholomew K, Kwong MJ, Hart SD (2001) Attachment. In: Livesley WJ (ed) Handbook of personality disorders: theory research and treatment. The Guilford Press, New York, pp 196-230

25. Meyer B, Pilkonis PA, Beevers CG (2004) What's in a neutral face? Personality disorders, attachment styles, and the appraisal of ambiguous social cues. J Pers Disord 18(4):320-336

26. Arntz A, Veen G (2001) Evaluations of others by borderline patients. J Nerv Ment Disord 189:513-521

27. Linehan MM (1993) Cognitive behavioural treatment for borderline personality disorder. Guilford Press, New York 